Pacific Journal of Mathematics

THE ASYMPTOTIC DISTRIBUTION OF THE
TIME-TO-ESCAPE FOR COMETS STRONGLY BOUND TO THE
SOLAR SYSTEM DAVID G. KeNDALL AND John Leonard MOtT 


\title{
THE ASYMPTOTIC DISTRIBUTION OF THE TIME-TO- ESCAPE FOR COMETS STRONGLY BOUND TO THE SOLAR SYSTEM
}

\author{
David G. Kendall and J. L. Mott.
}

1. Introduction This paper is one of a series (Hammersley and Lyttleton [1], [2], Kerr [3], Kendall [4], [5], [6]) concerned with the statistical-dynamical properties of the sun's family of comets. For the astronomical background, terminology, conventions, units, etc., we refer the reader to [5].

We consider a comet in the energy-state $x>0$ (so that the total energy per unit mass is equal to $-x$ ) which is approaching perihelion, not necessarily for the first time, and we write $T$ for the total time spent by the comet in describing complete circuits subsequent to this perihelion. We ignore the low energy (high $x$ ) catastrophes (capture by Jupiter, falling into the sun, etc.) and consider the fate of the comet subject to independent energy-perturbations at perihelion, the magnitudes of which we suppose to be distributed according to the probability law

$$
\frac{1}{2} e^{-|w| / b} d w / b \quad(-\infty<w<\infty),
$$

the so-called 'double-exponential law'. It is then known [5] that $T$ is almost certainly finite.

The probability distribution of $T$ cannot be found explicitly, but its Laplace-Stieltjes transform $\phi$ satisfies a differential equation which we treat by a perturbation method. At first sight it seems unlikely that a perturbation procedure followed by a Laplace inversion could yield any positive information about the distribution being studied, but in fact by a careful arrangement of the argument we are able to calculate the exact limit-law

$$
\lim _{x \rightarrow \infty} \operatorname{Pr}\left\{\frac{T}{\sqrt{x}} \leqq c \mid x\right\}
$$

for the reduced random variable $T / V x$; the result is given at (15) below.

If we are chiefly interested in the origin of comets we can identify the given perihelion with the comet's first, and $x$ is then its initial energy-state. There are indications ([5], [6]) that this value of $x$ is small when compared with the average size of the perturbations, but information about solutions for large $x$ can be extracted from Hammersley [2], and the present result thus forms a useful complement to some of his results, with which it is consistent: in fact, the same (limit-) law was obtained by Hammersley in his exact solution to the corresponding problem involv-

Received November 16, 1960. 
ing Brownian motion.

If we do not identify the given perihelion with the comet's first, then our result tells us the distribution of the remaining time-to-escape $T$ for a comet which happens to have entered a high energy-state. From this point of view the result is of value whatever opinion we may hold about the origin of comets, but it is of course limited by the fact that when $x$ is large (i.e., the comet is strongly bound) then one cannot properly neglect the low-energy (high $x$ ) catastrophes.

The justification for the use of the double-exponential perturbation law will be found in [3], [4], [5], [6]. Because of the asymptotic character of the present result one might expect the detailed form of the perturbation law to be unimportant, and one might hope that identically the same result would follow for any perturbation with zero mean and a finite variance. The proof that this is so is the object of an investigation by C. Stone and J. Lamperti, who will in a forthcoming paper discuss the appropriate invariance theorem.

In the course of our work we shall make use of some Bessel function formulae given by Watson [7]. We shall refer to these formulae as $(1 \mathrm{~W}), \cdots,(5 \mathrm{~W})$, where $(1 \mathrm{~W})$ is given on p. 80 of [7] at (19), (2W)-77(2), (3W)-202(1), (4W)-203(2) and (5W)-80(15).

2. The asymptotic distribution of $T / V x . \quad T$ is the total time during which a comet remains in the system, measured from (say first) perihelion; thus $T$ is the total time spent in describing complete circuits. The comet is subject to energy perturbations at perihelion distributed according to the double-exponential law, and there is also a chance $k(0 \leqq k<1)$ of disintegration at each perihelion passage; for the moment we retain the possibility of disintegration but our main results depend on a method which would not be very easy to handle when $k>0$, and we shall shortly put $k=0$.

Define

$$
\phi(s \mid x) \equiv \mathscr{E}\left(e^{-s T} \mid x\right)
$$

here $x$ is the energy-state of the comet during the approach to first perihelion, so that $x>0$, and $s \geqq 0 . \quad V(y)=y^{-3 / 2}(y>0)$ gives the periodic time of an orbit in state $y$, but (following Hammersley [2]) we shall first set $V(y)=y^{-\alpha}$. We shall later put $\alpha=3 / 2$ to give our main result, and afterwards remark briefly on the more general case.

Consideration of the possible events at first perihelion leads to the integral equation for $\phi$ :

$$
\begin{aligned}
\phi(s \mid x)=k & +(1-k)\left\{\frac{1}{2} e^{-x / b}+\int_{0}^{\infty} \frac{1}{2} b^{-1} e^{-w / b} e^{-s V(x+w)} \phi(s \mid x+w) d w\right. \\
& \left.+\int_{0}^{x} \frac{1}{2} b^{-1} e^{-w / b} e^{-s V(x-w)} \phi(s \mid x-w) d w\right\},
\end{aligned}
$$


whence

$$
\text { (1) } \begin{aligned}
\phi(s \mid x)=k & +(1-k)\left\{\frac{1}{2} e^{-x / b}+\frac{1}{2} b^{-1} e^{x / b} \int_{x}^{\infty} e^{-w / b} e^{-s V(w)} \phi(s \mid w) d w\right. \\
& \left.+\frac{1}{2} b^{-1} e^{-x / b} \int_{0}^{x} e^{w / b} e^{-s V(\omega)} \phi(s \mid w) d w\right\}
\end{aligned}
$$

Since $0 \leqq \phi \leqq 1$ for $x>0$ and $s \geqq 0$, and $\phi(s \mid \cdot)$ is measurable for $s \geqq 0$, we see from (1) that $\phi(s \mid \cdot)$ is continuous on the interval $0<x<\infty$. But then $\phi(s \mid \cdot)$ is also differentiable, and with $D \equiv \partial / \partial x$,

$$
\begin{aligned}
(1-k)^{-1} D \phi= & -\frac{1}{2} b^{-1} e^{-x / b}+\frac{1}{2} b^{-2} e^{x / b} \int_{x}^{\infty} e^{-w / b} e^{-s V(w)} \phi(s \mid w) d w \\
& -\frac{1}{2} b^{-2} e^{-x / b} \int_{0}^{x} e^{w / b} e^{-s V(w)} \phi(s \mid w) d w
\end{aligned}
$$

also

$$
\begin{aligned}
(1-k)^{-1} D^{2} \phi & =\frac{1}{2} b^{-2} e^{-x / b}+\frac{1}{2} b^{-3} e^{x / b} \int_{x}^{\infty} e^{-w / b} e^{-s \nabla(w)} \phi(s \mid w) d w \\
& -b^{-2} e^{-s \nabla(x)} \phi(s \mid x)+\frac{1}{2} b^{-3} e^{-x / b} \int_{0}^{x} e^{w / b} e^{-s \nabla(w)} \phi(s \mid w) d w
\end{aligned}
$$

Thus from (1) and (3)

$$
D^{2} \phi=b^{-2}\left\{1-(1-k) e^{-s V(x)}\right\} \phi(s \mid x)-k b^{-2} \quad(x>0) ;
$$

and now we have that, in fact, $\phi \in C^{\infty}$.

We now put $k=0$, and then write (4) as

$$
D^{2} \phi-g \phi=f \phi,
$$

where

$$
g=s b^{-2} V(x) \text { and } f=\left\{1-s V(x)-e^{-s \nabla(x)}\right\} b^{-2} .
$$

We discuss the nature of $\phi$ by using the standard method of variation of parameters, and so postulate as a solution of (5) (for the moment we suppress the variable $s$ )

$$
\phi(x)=A(x) \theta_{1}(x)+B(x) \theta_{2}(x),
$$

where $\theta_{1}(x), \theta_{2}(x)$ are independent solutions of

$$
D^{2} \phi-g \phi=0 \text {. }
$$

We find that

( 7$) \phi(x)=\theta_{1}(x) \int_{c}^{x} f(y) \phi(y) \theta_{2}(y) d y / W-\theta_{2}(x) \int_{a}^{x} f(y) \phi(y) \theta_{1}(y) d y / W$, where $W=\theta_{1}^{\prime}(y) \theta_{2}(y)-\theta_{2}^{\prime}(y) \theta_{1}(y)$ (actually a nonzero constant) and $c, d$ 
here are constants. When $\alpha=3 / 2$, which is the case we shall work through in detail, we can take (cf. Watson [7], p. 96)

$$
\theta_{1}(x)=x^{1 / 2} K_{2}\left(4 b^{-1} s^{1 / 2} x^{1 / 4}\right) \text { and } \theta_{2}(x)=x^{1 / 2} I_{2}\left(4 b^{-1} s^{1 / 2} x^{1 / 4}\right) ;
$$

also $W=-\frac{1}{4}$, using $(1 W)$. We now rewrite $(7)$ in the more convenient form:

$$
\begin{gathered}
\phi(x)=A \theta_{1}(x)+B \theta_{2}(x)-4 \theta_{1}(x) \int_{0+}^{x} f(y) \phi(y) \theta_{2}(y) d y \\
-4 \theta_{2}(x) \int_{x}^{\infty} f(y) \phi(y) \theta_{1}(y) d y
\end{gathered}
$$

where $A$ and $B$ are constants (possibly involving $s$ ) to be found later; this we can do because, for fixed $s, \theta_{2}(y) \sim C y(y \rightarrow 0)$ and $\theta_{1}(y) \sim$ $C y^{3 / 8} e^{-c y^{1 / 4}}(y \rightarrow \infty)$, by $(2 W)$ and $(3 W)$. Here (and elsewhere) $C$ is some positive constant (often depending on $s$ ), but not necessarily the same each time it occurs. Thus the effect of our work so far has been to replace the natural integral equation, (1), by a second, (9); but (9) is the easier to handle.

We now find $A$ and $B$. We first note that $B=0$. For

(i) $\theta_{1}(y) \sim C y^{3 / 8} \exp \left(-4 b^{-1} s^{1 / 2} y^{1 / 4}\right)(y \rightarrow \infty)$, by $(3 W)$,

(ii) $\theta_{2}(y) \sim C y^{3 / 8} \exp \left(4 b^{-1} s^{1 / 2} y^{1 / 4}\right)(y \rightarrow \infty)$, by $(4 W)$ and

(iii) $f(y)=O\left(y^{-3}\right)(y \rightarrow \infty)$; thus the two integral terms of (9) tend to zero as $x \rightarrow \infty$. Since $0 \leqq \phi \leqq 1$ for all $x$, there is (for $x \rightarrow \infty$ ) just one unbounded term in (9) if $B \neq 0$, and so $B=0$.

To find $A$ we need further boundary conditions on $\phi$. From (1) (with $k=0$ ) we have

$$
\phi(s \mid 0+)=\frac{1}{2}+\frac{1}{2} b^{-1} J,
$$

where

$$
J=\int_{0}^{\infty} e^{-w / b} e^{-s V(w)} \phi(s / w) d w ;
$$

and from (2) (with $k=0$ )

$$
D \phi(s \mid 0+)=-\frac{1}{2} b^{-1}+\frac{1}{2} b^{-2} J .
$$

Thus

$$
\phi(s \mid 0+)=1+b D \phi(s \mid 0+)
$$

which, with (9), allows us (after some detailed calculation) to evaluate $A$. We find, by elaboration of the methods used below, that

$$
A=\left\{\frac{b^{2}}{8 s}+\frac{s}{b} \log \left(\frac{4 s}{b^{2}}\right)+(2 \gamma-1) \frac{s}{b}\right\}^{-1} ;
$$


we shall not give the details because the asymptotic distribution of $T / V x$ is obtainable without this complete treatment of $A$. We shall show later (in $\S 4$ ) that

$$
\phi=A b^{2} / 8 s+O\left(x^{1 / 2}\right) \quad(x \rightarrow 0),
$$

when $s>0$, whence $\phi(s \mid 0+)=A b^{2} / 8 s$. Then from (10), recalling that $A$ depends on $s$, we see that

$$
\begin{aligned}
\lim _{s \rightarrow 0}\left(A b^{2} / 8 s\right) & =\frac{1}{2}+\frac{1}{2} b^{-1} \lim _{s \rightarrow 0} \int_{0}^{\infty} e^{-w / b} e^{-s V(w)} \phi(s \mid w) d w \\
& =\frac{1}{2}+\frac{1}{2} b^{-1} \int_{0}^{\infty} e^{-w / b} d w \operatorname{Pr}\{T<\infty\}=1
\end{aligned}
$$

(because almost certainly there will be only finitely many complete circuits), so that

$$
A \sim 8 s / b^{2} \quad(s \rightarrow 0) .
$$

Now put $s \sqrt{ } x=\sigma>0$ in (9) and let $x \rightarrow \infty$ and $s \rightarrow 0, \sigma$ being fixed. Then

$$
\mathscr{E}\left(e^{-\sigma T / \sqrt{ } x} \mid x\right)=\phi(s \mid x) \rightarrow 8 b^{-2} \sigma K_{2}\left(4 b^{-1} \vee \sigma\right),(\sigma>0, x \rightarrow \infty) .
$$

if both integral terms of (9) tend to zero; this is in fact the case, as we show in $\S 3$. The (honest) probability distribution

$$
\frac{16}{b^{4}} \exp \left(-\frac{4}{b^{2} \tau}\right) \frac{d \tau}{\tau^{3}}
$$$$
(0<\tau<\infty)
$$

has the expression on the right-hand side of (13) as its Laplace transform, so that

$$
\lim _{x \rightarrow \infty} \mathscr{E}\left(e^{-\sigma T / \sqrt{ } x} \mid x\right)=\mathscr{E}\left(e^{-\sigma \tau}\right)
$$

for all $\sigma>0$. It follows by the continuity theorem for the LaplaceStieltjes transforms of probability distributions of nonnegative random variables that

$$
\lim _{x \rightarrow \infty} \operatorname{Pr}\left\{\frac{T}{\sqrt{ } x} \leqq c \mid x\right\}=\frac{16}{b^{4}} \int_{0}^{c} \exp \left(-\frac{4}{b^{2} \tau}\right) \frac{d \tau}{\tau^{3}},=\left(1+\frac{4}{b^{2} c}\right) \exp \left(-\frac{4}{b^{2} c}\right) .
$$

This is our main result; but it is clear that (to some extent at least) the precise value of $\alpha$ affects the detail of (15) rather than its essential nature. For $\alpha \neq 2$ we can take as independent solutions of (9)

$$
\theta_{1}(x)=x^{1 / 2} K_{\nu}\left(2 \nu b^{-1} s^{1 / 2} x^{1-(1 / 2) \alpha}\right) \text { and } \theta_{2}(x)=x^{1 / 2} I_{\nu}\left(2 \nu b^{-1} s^{1 / 2} x^{1-(1 / 2) \alpha}\right),
$$

where $\nu=|\alpha-2|^{-1}$ (for $\alpha=2$ the solutions are powers of $x$ ), and then 
find as before that $W$ is a constant independent of $y$. But some limitations on $\alpha$ are imposed by the need for suitable behaviour of various integral terms, and we merely note here one analogue of (15):

if $V(x)=x^{-1}$, then

$$
\lim _{x \rightarrow \infty} \operatorname{Pr}\left\{\frac{T}{x} \leqq c \mid x\right\}=\frac{1}{b^{2}} \int_{0}^{c} \exp \left(-\frac{1}{b^{2} \tau}\right) \frac{d \tau}{\tau^{2}},=\exp \left(-\frac{1}{b^{2} c}\right) .
$$

3. Analytical details. Consider the behaviour of the integral terms in (9) when $x \rightarrow \infty$ and $s \sqrt{ } x=\sigma>0$. These terms are (apart from constant factors)

$$
\theta_{1}(x) \int_{0}^{x} f(y) \phi(s \mid y) \theta_{2}(y) d y
$$

and

$$
\theta_{2}(x) \int_{x}^{\infty} f(y) \phi(s \mid y) \theta_{1}(y) d y \text {. }
$$

Write (a) as $\theta_{1}(x)\left\{\int_{0}^{1}+\int_{1}^{x} f(y) \phi(s \mid y) \theta_{2}(y) d y\right\}=A_{1}+A_{2}$, say, and consider separately the terms $A_{1}$ and $A_{2}$. Using $|f|<2 b^{-2} s y^{-3 / 2}(y>0)$, and noting that $\theta_{1}(x)=\sigma s^{-1} K_{2}\left(4 b^{-1} \bigvee \sigma\right)$, we have

$$
\begin{gathered}
\left|A_{1}\right|<C \frac{\sigma}{s} \int_{0}^{1} \frac{s}{y^{3 / 2}} \cdot y^{1 / 2} I_{2}\left[\frac{4 \sqrt{ } \sigma}{b}\left(\frac{y}{x}\right)^{1 / 4}\right] d y \\
<C \int_{0}^{1} \frac{1}{y}\left(\frac{y}{x}\right)^{1 / 2} d y
\end{gathered}
$$

for $x$ sufficiently large, since $I_{2}\left(\lambda^{1 / 4}\right)=O\left(\lambda^{1 / 2}\right)(\lambda \rightarrow 0)$. Thus $A_{1}=O\left(x^{-1 / 2}\right)$ $(x \rightarrow \infty)$. For $A_{2}$ we use $|f|<\frac{1}{2} s^{2} / y^{3} b^{2}$ and find

$$
\left|A_{2}\right|<C \frac{\sigma}{s} \int_{1}^{x} \frac{s^{2}}{y^{3}} y^{1 / 2} I_{2}\left[\frac{4 \sqrt{ } \sigma}{b}\left(\frac{y}{x}\right)^{1 / 4}\right] d y<C s \int_{1}^{x} y^{-5 / 2} d y,
$$

since $I_{2}(\theta)$ is bounded for $0 \leqq \theta \leqq 4 b^{-1} V \sigma$. Thus $A_{2}=O\left(x^{-1 / 2}\right)(x \rightarrow \infty)$, so that (a) $\rightarrow 0$ as $x \rightarrow \infty$ with $s \sqrt{ } x=\sigma>0$.

For (b) have

$$
|(b)|<C x^{1 / 2} \int_{x}^{\infty} y^{1 / 2} \frac{s^{2}}{y^{3}} d y=O\left(x^{-2}\right) \quad(x \rightarrow \infty),
$$

which completes the proof that the integral terms tend to zero.

4. Analytical details (continued). We now prove (12). To do this we need (part of) 
(i) $\theta_{1}(x)=b^{2} / 8 s-\frac{1}{2} x^{1 / 2}+O(x \log x) \quad(x \rightarrow 0)$,

(ii) $\theta_{2}(x)=2 s b^{-2} x+O\left(x^{3 / 2}\right) \quad(x \rightarrow 0)$

and

(iii) $1-s V(x)-e^{-s V(x)}=-s x^{-3 / 2}+O(1) \quad(x \rightarrow 0)$.

Proofs. By $(5 W)$,

$$
K_{2}(z)=2 z^{-2}-\frac{1}{2}+O\left(z^{2} \log z\right) \quad(z \rightarrow 0),
$$

whence (i) follows. Likewise, using ( $2 \mathrm{~W}$ ), (ii) follows from

$$
I_{2}(z)=z^{2} / 8+O\left(z^{4}\right) \quad(z \rightarrow 0) .
$$

Finally, (iii) follows from $0<e^{-s V(x)}<1$ for $x>0, s>0$.

We now note that (12) follows at once from (9) if we show that

$$
\text { (iv) } \theta_{1}(x) \int_{0+}^{x} f(y) \phi(y) \theta_{2}(y) d y=O\left(x^{1 / 2}\right) \quad(x \rightarrow 0)
$$

and

$$
\text { (v) } \theta_{2}(x) \int_{x}^{\infty} f(y) \phi(y) \theta_{1}(y) d y=O\left(x^{1 / 2}\right) \quad(x \rightarrow 0) .
$$

These results follow from those already given. We have

$$
f(y)=O\left(y^{-3 / 2}\right)(y \rightarrow 0) \text { and } \theta_{2}(y)=O(y)(y \rightarrow 0)
$$

so that

$$
\int_{0}^{x} f(y) \phi(y) \theta_{2}(y) d y=O\left(x^{1 / 2}\right) \quad(x \rightarrow 0) .
$$

Since $\theta_{1}(x)=O(1)(x \rightarrow 0)$, this gives (iv). The proof of $(\mathrm{v})$ is similar.

\section{REFERENCES}

1, 2. J. M. Hammersley and R. A. Lyttleton, On the statistical loss of long period comets from the solar system, I, II, 4th Berkeley Symp. on Math. Stat. and Prob., Berkeley, University of California Press, 1961.

3. R. H. Kerr, Perturbations of cometary orbits, 4th Berkeley Symp. on Math. Stat. and Prob., Berkeley, University of California Press, 1961.

4. D. G. Kendall, The distribution of energy perturbations for Halley's and some other comets, 4th Berkeley Symp. on Math. Stat. and Prob., Berkeley, University of California Press, 1961.

5, 6. D. G. Kendall, Some problems in the theory of comets, I, II, 4th Berkeley Symp. on Math. Stat. and Prob., Berkeley, University of California Press, 1961.

7. G. N. Watson, A treatise on the theory of Bessel functions, second edition, Cambridge, 1944.

Magdalen College, OXFord.

UNIVERSITY OF EDINBURGH. 



\section{PACIFIC JOURNAL OF MATHEMATICS}

\section{EDITORS}

\author{
RaLPh S. Phillips \\ Stanford University \\ Stanford, California \\ F. H. BRowNELL \\ University of Washington \\ Seattle 5 , Washington
}

A. L. Whiteman

University of Southern California

Los Angeles 7, California

L. J. Paige

University of California

Los Angeles 24, California

\author{
E. F. BECKENBACH \\ T. M. CHERRY
}

\author{
ASSOCIATE EDITORS

$\begin{array}{lll}\text { D. DERRY } & \text { H. L. ROYDEN } & \text { E. G. STRAUS } \\ \text { M. OHTSUKA } & \text { E. SPANIER } & \text { F. WOLF }\end{array}$

\section{SUPPORTING INSTITUTIONS}

\author{
UNIVERSITY OF BRITISH COLUMBIA \\ CALIFORNIA INSTITUTE OF TECHNOLOGY \\ UNIVERSITY OF CALIFORNIA \\ MONTANA STATE UNIVERSITY \\ UNIVERSITY OF NEVADA \\ NEW MEXICO STATE UNIVERSITY \\ OREGON STATE COLLEGE \\ UNIVERSITY OF OREGON \\ OSAKA UNIVERSITY \\ UNIVERSITY OF SOUTHERN CALIFORNIA
}

\author{
STANFORD UNIVERSITY \\ UNIVERSITY OF TOKYO \\ UNIVERSITY OF UTAH \\ WASHINGTON STATE COLLEGE \\ UNIVERSITY OF WASHINGTON \\ AMERICAN MATHEMATICAL SOCIETY \\ CALIFORNIA RESEARCH CORPORATION \\ HUGHES AIRCRAFT COMPANY \\ SPACE TECHNOLOGY LABORATORIES \\ NAVAL ORDNANCE TEST STATION
}

Mathematical papers intended for publication in the Pacific Journal of Mathematics should be typewritten (double spaced), and the author should keep a complete copy. Manuscripts may be sent to any one of the four editors. All other communications to the editors should be addressed to the managing editor, L. J. Paige at the University of California, Los Angeles 24, California.

50 reprints per author of each article are furnished free of charge; additional copies may be obtained at cost in multiples of 50 .

The Pacific Journal of Mathematics is published quarterly, in March, June, September, and December. The price per volume (4 numbers) is $\$ 12.00$; single issues, $\$ 3.50$. Back numbers are available. Special price to individual faculty members of supporting institutions and to individual members of the American Mathematical Society: $\$ 4.00$ per volume; single issues, $\$ 1.25$.

Subscriptions, orders for back numbers, and changes of address should be sent to Pacific Journal of Mathematics, 103 Highland Boulevard, Berkeley 8, California.

Printed at Kokusai Bunken Insatsusha (International Academic Printing Co., Ltd.), No. 6, 2-chome, Fujimi-cho, Chiyoda-ku, Tokyo, Japan.

\section{PUBLISHED BY PACIFIC JOURNAL OF MATHEMATICS, A NON-PROFIT CORPORATION}

The Supporting Institutions listed above contribute to the cost of publication of this Journal, but they are not owners or publishers and have no responsibility for its content or policies.

Reprinted 1966 in the United States of America 


\section{Pacific Journal of Mathematics}

\section{Vol. 11, No. 4}

A. V. Balakrishnan, Prediction theory for Markoff processes . . . . . . . . . . 1171

Dallas O. Banks, Upper bounds for the eigenvalues of some vibrating systems . . . . 1183

A. Białynicki-Birula, On the field of rational functions of algebraic groups ...... 1205

Thomas Andrew Brown, Simple paths on convex polyhedra .............. 1211

L. Carlitz, Some congruences for the Bell polynomials . . . . . . . . . . . . 1215

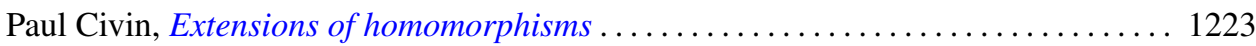

Paul Joseph Cohen and Milton Lees, Asymptotic decay of solutions of differential

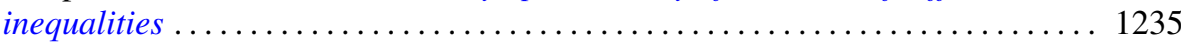

István Fáry, Self-intersection of a sphere on a complex quadric . . . . . . . . . . 1251

Walter Feit and John Griggs Thompson, Groups which have a faithful representation

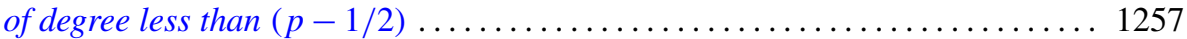

William James Firey, Mean cross-section measures of harmonic means of convex

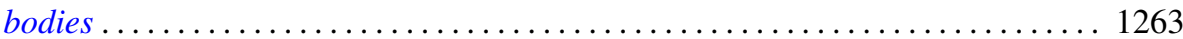

Avner Friedman, The wave equation for differential forms . . . . . . . . . . 1267

Bernard Russel Gelbaum and Jesus Gil De Lamadrid, Bases of tensor products of

Banach spaces ................................... 1281

Ronald Kay Getoor, Infinitely divisible probabilities on the hyperbolic plane . . . . 1287

Basil Gordon, Sequences in groups with distinct partial products . . . . . . . . . . . . 1309

Magnus R. Hestenes, Relative self-adjoint operators in Hilbert space . . . . . . . . . 1315

Fu Cheng Hsiang, On a theorem of Fejér ......................... 1359

John McCormick Irwin and Elbert A. Walker, On N-high subgroups of Abelian

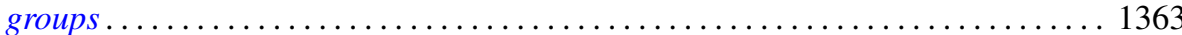

John McCormick Irwin, High subgroups of Abelian torsion groups . . . . . . . . . 1375

R. E. Johnson, Quotient rings of rings with zero singular ideal . . . . . . . . . . . 1385

David G. Kendall and John Leonard Mott, The asymptotic distribution of the time-to-escape for comets strongly bound to the solar system ...

Kurt Kreith, The spectrum of singular self-adjoint elliptic operators ....

Lionello Lombardi, The semicontinuity of the most general integral of the calculus of variations in non-parametric form ................................

Albert W. Marshall and Ingram Olkin, Game theoretic proof that Chebyshev inequalities are sharp

Wallace Smith Martindale, III, Primitive algebras with involution . . William H. Mills, Decomposition of holomorphs ..............

James Donald Monk, On the representation theory for cylindric algebras . . . . . . 1447

Shu-Teh Chen Moy, A note on generalizations of Shannon-McMillan theorem . . . . 1459

Donald Earl Myers, An imbedding space for Schwartz distributions . .

John R. Myhill, Category methods in recursion theory .........

Paul Adrian Nickel, On extremal properties for annular radial and circular slit mappings of bordered Riemann surfaces

Edward Scott O'Keefe, Primal clusters of two-element algebras . .

Nelson Onuchic, Applications of the topological method of Wazewski to certain

problems of asymptotic behavior in ordinary differential equations ...

Peter Perkins, A theorem on regular matrices................

Clinton M. Petty, Centroid surfaces .... 